ESTUDIOS 


\section{MOTIVO Y SIGNIFICADO DE LA CORONACIÓN DE PEDRO II DE ARAGÓN.}

por

\section{DAMIÁN SMITH}

Leverhulme study abroad student

C.S.I.C., Barcelona*

RESUMEN: En la relación entre el papado y la Corona de Aragón, la coronación del Rey Pedro II (1196-1213) por el Papa Inocencio III (1198-1216), fiesta de San Martín 1204, era, sin duda, un acontecimiento muy importante. No obstante, tadavia bay muchas preguntas complicadas aceria de la ceremonia y de sus motivaciones. Este estudio aumenta nuestra información sobre la coronación en Roma, los motivos del Rey y del Papa, y sugiere la trascendencia a largo plazo de la coronación.

Palabras CIAVE: Corona de Aragón, papado, siglos XII y XIII, coronaciones, Inocencio III, Pedro II.

\footnotetext{
* Agradezco al Dr. Ignacio Álvarez Borge y al Sr. John Doran por su inestimable ayuda. La investigación para éste artículo fue apoyada por la concesión de la beca Francis Corder Clyton de la Universidad de Birmingham.

Además de las abreviaturas estándar, se usarán las siguientes:

ACA $=$ Arxiu de la Corona d'Aragó.

MDbI= La documentación pontificia hasta Inocencio III (965-1216), ed. Demetrio MANSILLA, RoMA 1955.

Miquel= Regesta de Letras Pontificias del Archivo de ha Corona de Aragón, ed. Francisco MiQUEL ROSELl, Madrid, 1948.

Potthast = Volumen I de Regesta Pontificum Romanorum inde ab anno 1198 ad annum 1304, ed. Augustus POTTHAST, 2 vols., Berlín 1874-1875.

Register= Die Register Innocenz 'II, vol 1 (1198/1199), 2 (1199/1200), 5 (1202/ 1203), 6 (1203/1204), 7 (1204/1205), ed. Otbmar HAGENDER, A. HAIDACHER, Wemer MALECZEK, Alfred STRNAD, Andrea SOMMERLECHNER, Henvig WEIGL, Cbristoph EGGER, Jobn MOORE, Rainer MURAUER, Karl RuDolf, Graz-Colonia-Roma-Viena, 1964-1997.
}

Hispania, LX/1, núm. 204 (2000) 163-179 
ABSTRACT: In the relations bip between the papacy and the Crown of Aragon, the coronation of Peter II (1196-1213) by Pope Innocent III (1198-1216) on the Feast of St Martin 1204 was undoubtedly a very important event. Still, bowever, there remain many complicated questions concerning the ceremony and the reasons bebind it. This anticle attempts to add information on the coronation in Rome, the political motives of the King and the Pope, and to suggest the long-term significance of the coronation.

KEY words: Crown of Aragon, Papacy, Twelfth and Thirteenth Centuries, Coronation, Innocent III, Peter II.

En la larga relación entre Roma y Aragón-Cataluña, pocos acontecimientos fueron más trascendentales, más complejos en sus motivos, más significantes en sus efectos perdurables, que la coronación de Pedro II (1196-1213) en Roma el 11 de noviembre de 1204 .

Este estudio, una pequeña contribución a las celebraciones del aniversario del advenimiento del Papa Inocencio III (1198-1216), pretende:

1) Clarificar ciertas cuestiones acerca de la propia coronación en 1204.

2) Situar la coronación en su contexto histórico y analizar los posibles motivos de ambas partes, Aragón y el Papado.

3) Ofrecer sugerencias más amplias sobre el significado histórico del acontecimiento.

Se espera que este artículo especulativo, cuya intención no es la radical reinterpretación de la coronación de Pedro, pueda añadir nueva información respecto a tal hecho y servir de plataforma para estudios posteriores.

1. Conocemos la coronación con cierto detalle a través del Ordo Coronationis Petri regis Aragonum ${ }^{\mathrm{I}}$, conservado en la chancillería Papal, y también copiado de ahí por el autor anónimo de Gesta Inocentii Papae $1 I^{2}$, una biografía contemporánea del Papa. El Ordo ya no existe en la colección de correspondencia papal-real en el Archivo de la Corona de Aragón en Barcelona ${ }^{3}$, aunque este documento o una copia, evidentemente es posible que lo consultaran en el siglo XVI o principios del XVII Blancas y Zurita, historiadores pioneros de Aragón ${ }^{4}$. 550.

' Register 7, $\mathrm{n}^{\circ}$ 229, pp. 406-409; MDbI, n'. 307, pp. 339-341; Potthast, 2322, 2361; PL, 215 ,

2 Gesta Inocentii III, c. 120-122 (PL, 214, 159-161).

3 Para las bulas de Inocencio III existentes en el ACA, ver Miquel, pp. 39-51, números 44-69.

1 BLANCAS, Jerónimo de, Coronaciones de los serenissimos Reyes de Aragón (c. 1585), Zaragoza 1641, pp. 1-15; ZuRuTs, Gerónimo, Anales de la Corona de Aragón, ed. Antonio Ubieto Arteta, 4 vols., Valencia 1967,2 , pp. 140-143.

Hispania, LX/1, núm. 204 (2000) 163-179 
1.1. La existencia del Ordo era más notable dado que ni Roma ni Zaragoza, Tatragona ni Huesca poseían un Ordo para la coronación de un Rey. Los Reyes simplemente no eran coronados en Roma, ni (al menos con ninguna ceremonia fija) en Aragón's. En ausencia de un Ordo parece que, por la forma del ceremonial, este fue una adaptación de la del advenimiento papal, del Ordo Romano para la coronación imperial, del Ordo de coronación de la Iglesia de Arles y de la ceremonia de coronación Papal para el día de San Martín, con motivo de esta ocasión única, aunque algunos elementos fueran $a d b c^{6}$.

1.2. ¿Qué nos dice el Ordo? El 9 de noviembre, cinco galeras de Pedro II atracaron en una isla entre Porto y Ostia en el río Tíber. Pedro estaba acompañado por un gran número de religiosos y nobles, de los cuales el Ordo menciona específicamente al Arzobispo Michael de Arles, al preboste de Maguelonne (Guy de Ventadour), al electo de Montemaior (William de Bonnieux), al Conde Sancho (tío de Pedro), a Hugh de Baux, a Rocelin de Marseille y a Arnau de Castellbó. Inocencio III envió a Pedro un escuadrón de caballería y puso a ciertos Cardenales, al senador de la Ciudad, y a muchos nobles romanos a disposición del Rey. Pedro se presentó ante Inocencio III y fue huésped de honor de los canónigos de San Pedro.

1.3. El 11 de noviembre, fiesta de San Martín, Inocencio y su séquito, con muchos nobles y gente, fueron en procesión al monasterio de San Pancracio. San Pancracio tenía un tamaño apropiado y estaba a una distancia aceptable desde San Pedro, extramuros (con lo cual no cedía derechos jurisdiccionales ni removía recuerdos en los Tarquinios) y quizá tiene el valor añadido de tratarse de una basílica y un santo muy asociados con los juramentos ${ }^{7}$. El Rey Pedro fue ungido (debió de serlo en los brazos y hombros, habiendo insistido Inocencio en que el ungimiento de la cabeza, con óleos consagrados estaba reservado a los obispos) ${ }^{8}$

5 Ante la falca de un Ordo de coronación Romana, ver WILKs, Michael, The problem of Sovereignity in the Later Middle ages: The Papal Monacby with Augustinus Triumpbus and the Publicist, Cambridge 1963, p.423; para un debate de la escasa evidencia de una coronación de Alfonso I en 1106, ver el excelente estudio de Palacios MARTín, Bonifacio, La coronación de los Reyes de Aragón, 12041410: Aportación al estudio de las estructuras politicas medievales, Valencia, 1975, pp. 21-23.

6 Para la coronación del Emperador, ver Ordo Romanus XII (PL 78, 1063-1106). Para el Ordo de Arles, Andrieu, M., Le Pontifical Romain III. Le Pontifical de Guillaume Durand, Vaticano, 1940, pp. 318-320; SChramm, Percy, «Die Krönung in Deutschland bis zum Beginn des Salisches Hauses (1228)»: Zeitschrift der Savigny-Stiffung für Rechtsgeschicbte 24 (1935) I84-332. Para el acto de llevar la corona papal, ver Eichmann, E., Weibe und Könung des Papstes im Mittelalter, Munich 1951, pp. 40-41. Sobre el adventus papal, ver TWYMAN, Susan, «Papal adventus at Rome in the Twelfth Century": Bulletin of the Institute of Historical Research 69-70 (1996), 233-253.

? GREGORY OF TOURS, De gloria martyrum, c. 38 en MGH, script. rer, merov, 1, p. 512; KRaut-Heimer, R, The Early Cbrittian Basilicas of Rome (IV-lXc), 3 vols., Vatican City 1976, 3 , Pp. 153- 174; SCHRAMM, «Die Kronung in katalanisch-aragonesischen Königreich»; Kaiser, Könige und Päpste: Gesammelte Ausfsätze zur Geschichte des Mitterlalters IV, 4 vols., Stuttgart 1970, 1, p. 355.

в (25/2/1204) «Cum venisset», Register 7, ภ० 3, pp. 8-13; Potthast 2138; PL, 215, 282-287. Ver KANTORowICZ, Ernst, The King's two bodies: A Study in Mediaeval Political Theology, Princecon, 
por Pedro, cardenal obispo de Porto (más que cardenal obispo de Ostia, quizá para distinguirlo de la coronación imperial, quizá porque San Pancracio estaba en el área administrativa de Porto) $)^{9}$ y coronado por el Papa, que le dio el manto, la cota, el cetro, el orbe, la corona y la mitra. Pedro hizo sobre los Evangelios el juramento de la coronación: que tendría siempre fe y obediencia al Papa y a la Iglesia Romana, que defendería el Catolicismo y perseguiría la herejía, guardaría la libertad de la Iglesia y defendería sus leyes y serviría a la justicia y a la paz en todas sus tierras ${ }^{10}$.

1.4. El Rey coronado, para celebrar una cálida recepción, marchó con Inocencio a San Pedro, y allí, sobre el altar, el Rey Pedro colocó su cetro y su corona. Pedro recibió entonces su espada de caballero de la mano de Inocencio"1. Pedro le dio a éste un documento en el que, titulado «Rey de los Aragoneses, Conde de Barcelona y Señor de Montpellier», reconocía la autoridad del Romano Pontífice como sucesor de San Pedro y Vicario de Cristo, y ofrecía su reino a Inocencio y a la Sede Apostólica, prometiendo pagar al Papa y a sus sucesores, a perpetuidad, un censo de 250 mazmudines de la cámara del Rey en compensación por la protección papal ${ }^{12}$. Inocencio escoltó a Pedro a través de la ciudad hasta la basílica de San Pablo y, hallando sus galeras preparadas, Pedro partió con la bendición del Papa ${ }^{13}$.

1957, pp. 319-320; BLOCH, Marc, The Royal Touch, Londres, 1973, pp. 85; UlLMANN , Walter, The Growth of Papal Government in the Middle Ages, Londres, 1962, pp. 227-278.

9 MALECZEK, Werner, Papst und Kardinalskolleg von 1191 bis 1216, Viena, 1984, pp. 80-83, 383; EichmanN, Weibe and Krönung des Papstes, Munich, 1951, pp. 50 1; Ullmann, Grouth of Papal government, p. 226.

${ }_{10}$ Register 7, n 229, p. 408: «Ego Petrus, rex Aragonum, profiteor et polliceor, quod semper ero fidelis et obediens domino pape Innocentio eiusque catholicis successoribus et ecclesie Romane regnumque meum in ipsius obedientia fideliter conservabo, defendens fidem catholicam et persequens herecicam pravitacem. Libertatem et immunitatem ecclesiarum custodiam et earum iura defendam. In omni terra mee potestati subiecta iustitiam et pacem servare studebo, sic me Deus adiuver et hec sancta Evangeliaw.

11 «Deinde prefatus rex cum multo laudis preconio et favoris applausu coronatus rediit iuxta dominum papam ad basilicam sancti Petri, super cuius altare sceptrum et diadema deposuit, et de manu eiusdem domini pape militarem ensem accepit.»

12 *Cum corde credam et ore confitear, quod Romanus pontifex, qui est beati Petri successor, vicarius sit illius, per quem reges regnant et principes principantur, qui dominatur in regno hominum et, cui voluerit, dabit illud. Ego Petrus, Dei gratia rex Aragonum, comes Barchinonie et dominus Montis Pesulani, cupiens principali post Deum beati Petri et apostolice sedis protectione murniri, tibi, reverentissime pater et domine summe pontifex Innocenti, et per te sacrosancte Romane apostolice sedo offero regnum meum illudque tibi et successoribus tuis in perpetuum divini amoris intuitu et pro remedio anime mee et progenitorum meorum constituo censuale, ut annuatim de camera regis ducente quinquaginte Massemutine apostolice sedi reddantur et ego ac successores mei specialiter ei fideles et obnoxii teneamurs.

${ }_{13}$ aHiis omnibus rite peractis fecit eum dominus papa per Vrbern ad ecclesian sancti Pauli deduci, ubi galeas inveniens preparatas intravit et apostolica benedictione munitus ad propria meruit cum prosperitate redire».

Hispania, LX/1, núm. 204 (2000) 163-179 
2. ¿Por qué se coronó Pedro en Roma? Las fuentes medievales nos dejan mucho trabajo por hacer. En una carta del 8 de agosto de 1204, Inocencio aseguraba a Pedro que él abandonaría Roma «con un incremento en su honor real» ${ }^{14}$. El 8 de octubre de 1204, en Marsella, Pedro II explicaba que había hecho su testamento edeseando visitar el umbral de los santos apóstoles Pedro y Pablo»15; el Ordo Coronationis declara que Pedro vino a Roma a recibir del Papa el cinturón de la caballería y la diadema real ${ }^{16}$; el Gesta Comitum Barcinonensium, cuya sección concerniente al reino de Pedro es casi - y probablemente- contemporánea, afirma: «El Rey Pedro rehusaba tener menos valentía o nobleza que sus predecesores y quería excederlos, deseando fama y reputación ${ }^{17}$; el Arzobispo Rodrigo Jiménez de Rada, que conocía bien a Pedro, en De Rebus Hispaniae (c. 1243), sólo dice que Pedro fue a Roma y fue coronado por el Papa en San Pancracio - no más, si bien es cierto que el acontecimiento no aumentó la gloria de Toledo ${ }^{18}$; La Crónica de San Juan de la Peña, la historia oficial aragonesa (hacia 1370), habla del deseo del Rey de «acrecentar la fama de su nombre» ${ }^{19}$.

14 ACA, Butlles Innocent III, leg. 3, $\mathrm{n}^{\circ}$ 7; ed. FRIED, Johannes, Der Päpstlicbe Schutz fïr Latenfürsten: Die politiscbe Geschichte des päpstlichen Schutzprivilegs für Laien (11-13. Jh), Heidelberg 1980, p. 329; cited Miguel, $\pi^{\circ} 51$, p. 42; $M D b l, n^{\circ} 306$, p. 339: 'Visum est, autem, nobis diligentius intuentibus, quod cum de gratia nostra dubitare non possis, utiliati tue magis expediat, ut ad presentiam nostram recto cursu procedas obtenturus a nobis, que desiderat anima tua, sicque consilio nostro diligenter instructus cum regalis honoris augmento apostolice sedis favore revertens, apud Pisanos et Ianuenses quod intendis facilius obtinebis mediante legato nostro, quem tecum auctore domino transmittemus',

15 Mirer I SANS, Joaquín, "Itinerario del rey Pedro I de Cataluña, II de Aragón»: Boletin de La Real Academia de Buenas Letras de Barcelona 3 (1905-6) 284; Histoire générale de Languedoc, ed. Joseph VAISSìte and Claude Devic, 16 vols., Toulouse 1872-1893, 4, p. 239: avolens visitare limina beatorum apostolorum Petri et Paulin.

16 Register 7, $n^{\circ} 229$, p. 407: 'Anno septimo pontificatus domini Innocentii tertii pape, mense Novembris, Petrus, rex Aragonum, ad apostolicam sedem accessit, ut ab eadem domino papa militare cingulum et regium acciperet diadema'.

17 Gesta Comitum Barcinonensium, ed. Louis Barrau DiHIGo and J. Massó TorrenTS, Barcelona 1925, c. 25, p. 51: 'Er quia praedictus dominus rex Petrus noluir probizate et nobilirate inferior praedecessoribus suis esse, immo antecellere eos cupiens fama et dignitate, Apostolorum limina cum multis sumptibus et comitatu nobili visiravit'; on the Gesta, see, BIsson, Thomas, «Unheroed Pasts: History and Commemoration in Souch Frankland before the Albigensian Crusades»: Speculum 65 (1980) 306-307.

IB Sobre el valor de Rodrigo JIMÉNEZ DE RADA como historiador, ver Historia de rebus Hispanie sive Historia Gotbica, Bk VI, ch. 4, p. 181: 'Hic rex Petrus sub Inocencio Papa III Romam adiens, ab eodem Papa fuit in ecclesia Sancti Pancracii diademathe coronacus'. Véase también LomAX, Derek, «Rodrigo Jiménez de Rada como historiador»: Actas del Quinto Congreso Internacional de Hispanistas, Bordeaux 1977, 587-592. Sobre la coronación de Pedto y Toledo, ver LiNehan, Peter, History and the Historians of Medieval Spain, Oxford 1994, pp. 389-390.

19 Véase La Crónica de San Juan de la Peña, ed. y trad., Lynn Nelson, University of Penssylvania 1991, c. 34, p. 57. También ver aquí Pere TOMICH, Histories e Conquestes dels Reys d"Arago e Comtes de 
No se puede cuestionar el deseo de peregrinación ${ }^{20}$; ni tampoco que Pedro deseara emular a sus antepasados, que desde el conde catalán Sunifredo II en el $951^{21}$ y el rey aragonés Sancho Ramírez en $1068^{22}$, habían realizado el camino de peregrinaje a Roma; ni tampoco se puede dudar del deseo de gloria de Pedro. Poco más hizo en su vida que perseguirla ${ }^{23}$. Si bien otros gobernantes gustaban de la peregrinación, tuvieron asociaciones directas con Roma y consiguieron gloria, no fueron coronados por el Papa en Roma. ¿Y por qué en 1204 ? Pues este acto de legitimación de la monarquía, este acontecimiento glorificador, tuvo que asociarse con una serie de circunstancias políticas peculiares. Se han dado muchas explicaciones; muchas de ellas son plausibles; con todas hay problemas, interrogantes; ninguna es satisfactoria individualmente; todas pueden combinarse; y quizá lo más plausible es no dar una preeminencia decisiva a ninguna.

2.1.1. Sicilia. Pedro II pretendía el matrimonio de su hermana Sancha con el rey Federico de Sicilia, buscando prestigio para ser merecedor ante los Staufer. Cuatro cartas de Inocencio III indican que este problema fue discutido en la coronación ${ }^{24}$. Para Pedro, las ventajas dinásticas y económicas de una posición establecida en Sicilia podían ser grandes. En apoyo al matrimonio, Pedro ofrecía ayuda financiera y militar a Federico. Realmente Pedro envió ayuda en dos ocasiones ${ }^{25}$. Y éste es el problema. La proposición inicial de alianza matri-

Catalunya, Valencia 1970 (facsimile of 1534 edition), c. 38, f. 32, p. 77; JALME DOMENECH, Crónica, Valencia 1975 (orig. 1380), c. 39, p. 46.

20 Sobre los aspectos de peregtinación de larga duración, JOSEPH GUDIOL, Mossèn, «De peregrinis i peregrinatges religiosos Catalans»: Analecta Sacra Tarraconensia 3 (1927), 93-119; Para los de corta duración, UBIETo ARTETA, Antonio, «la peregrinación de Alfonso II de Aragón a Santiago de Compostelay: Estudios de la Edad Media de la Corona de Aragón (EEMCA) 5 (1952), pp. 438-452.

21 Ver Abadal I de VinYaLs, Ramon, aL'esprit de Cluny i les telacions de Catalunya amb Roma i Itàlia al segle X»: Studi Medievali, Spoleto 1961, p. 14.

22 Ver KEHR, Paul, "Cómo y cuándo se hizo Aragón feudatorio de la Santa Sede»: EEMCA 1 (1945) 302; KeHR, "El Papado y los reinos de Navarra y Aragón hasta mediados del Siglo XIl»: EEMCA 2 (1946) 94-95.

23 Para el reinado de Pedro II en general, ver: Ventura Subirats, Jordi, Pere el Catòlic I Sirnò de Montfort, Barcelona 1960, y BAGUE, Enric, «Pere el Catòlic»: Els Primers Comtes-Reis: Ramon Berenguer IV, Alfons el Cast, Pere el Catdlic, ed. P. SCHRAMm, Barcelona 1963, pp. 103-145.

24 'Gaudemus in Domino' (8/8/1204), ACA, Butlles, Innocent III, leg. 3, $\mathrm{n}^{\circ} 7 ; \mathrm{MD}$, $\mathrm{n}^{\circ} 306$, p. 339; Miquel, nं 51 , p. 42; FR'ED, Päpstlicbe Scbutz, p. 329. 'Dilectum filium' (24/11/1204), ACA, Butlles, Innocent III, leg. 3, $\mathrm{n}^{\circ}$ 8; MDhI, $\mathrm{n}^{\circ}$ 308, pp. 341-2; Miquel, $\mathrm{n}^{\circ}$ 52, p. 43. 'Super negotio' (2/11/1207), ACA, Butlles, Innocent III, leg. 3, no 14; MDbI, n 374, p. 395; Miquel, no 58, p. 45. 'Ex speciali dilectionis' (25-7/2/1208), MDhI, $\mathrm{n}^{\circ}$ 382, p. 398; Potthast, 3306-3307; Miquel, $\mathrm{n}^{\circ}$ 59, p. 45.

25 «Ex speciali dilectionis' (25-7/2/1208) MDbI, $\mathrm{n}^{\mathrm{0}}$ 382, p. 398; Posthast, 3306-7; PL, 215, 1342. 'Gaudemus in Domino' (28/3/1209), ACA, Butlles, Innocent III, leg. $3, \mathrm{n}^{\circ} 17 ; \mathrm{MD}$, $\mathrm{n}^{\circ}$ 399, pp. 416.417; Miquel, $\mathrm{n}^{\circ}$ 61, p. 46. Sobre los intereses catalanes en el Mediterráneo, BoNNASSIE, Pierre, La Cazalogne du milieu du Xe à la fin du Xle siècle, 2 vols, Toulouse 1975-1976, 2, p. 842; DufourQ, Ch.-E., and Gautier Dalchí, Jean, Histoire économique et sociale de l'Espagne chré- 
monial vino por parte de la Emperatriz Constanza en $1198^{26}$. El matrimonio de Federico y la hermana de Pedro, Constanza, viuda de Imre de Hungría desde el 30 de noviembre de 1204, tuvo lugar en Palermo en otoño de $1209^{27}$. Si ocasionalmente Pedro mostró entusiasmo por el matrimonio, en más ocasiones dudó, en parte por razones financieras, y en parte a causa de la inestabilidad de la posición de Federico, e Inocencio a menudo le reprendió por esto ${ }^{28}$. Tomados en conjunto, las acciones de Pedro no sugieren que deseara especialmente ser digno de los Staufer.

2.1.2. Mallorca. Para ganar apoyo internacional para la conquista de Baleares. Tres cartas Papales de junio de 1205, estableciendo provisionalmente una sede episcopal en Mallorca ${ }^{29}$, urgiendo la paz entre Navarra y Castilla ${ }^{30}$, dando instrucciones a Calatrava y Alcántara para ayudar a Pedro en la guerra contra los musulmanes ${ }^{31}$, justifican que éste problema fuera tratado en Roma. Antes de la coronación, Pedro fue a Génova para ganar apoyo para su empre$\mathrm{sa}^{32}$. La conquista de Mallorca, un centro de comercio internacional, fue una ambición de los catalanes largamente sostenida y su captura se convirtió en un asunto particular después de la exitosa invasión por los almohades en $1203^{33}$.

tienne au Moyen Âge, Paris 1976, p. 105; DuFourQ, «Les Catatans, la méditerranée et la Sicite»: Annales 23 (1968), pp. 1120-1123; CARrÈrE, Claude, "Marseille, Aigues-Mortes, Barcelone, et la competition en Méditerranée au XIIIe siècle»: Antuario de Estudios Medievales 10 (1980), pp. 161. 172; ARTizzU, Francesco, "Penetrazione Cacalana in Sardegna nel secolo XII": Congreso de Historia de la Corona de Aragón [CHCA], 6 (1959), 87-99; FERrer I MALLOL, Teresa, "Els Italians a terres Catalanes (segles XII-XV)", pp. 393-467, and BATLLE I GALLART, Carme, «Els Francesos a la Corona d'Aragó", pp. 361-392: La Península Ibérica y El Mediterráneo centro-occidental (siglos XII-XV), Barcelona 1980; VOLPE, G., Studi sulle istituzioni communali a Pisa, 1970, pp. 166-167, 225.

26 Register 5., p. 98, nota 5; ABULAfIA, David, Frederick II; A Medieval Emperor, London 1992, p. 92; VAN Cleve, Thomas, The Emperor Frederick II of Hobenstaufen: Immutator Mundi, Oxford 1972, pp. 48-49; KANTOROWICZ, Enst, Frederick the Second 1194-1250, London 1931, p. 31.

27 Recueil des Actes des Contes de Provence appartenant a la maison de Barcelone. Alpbonse II et Raimond Bérengzer V (1196-1245), ed. F. BENOIT, $\mathrm{n}^{\circ} 66$; ABULAFIA, Frederick II, p. 106; VAN CleVE, Emperor Frederick II, pp. 65-66; KANTOROWICZ, Frederick the Second, pp. 34-35.

28 'Super negotio' ACA, Butlles, Innocent III, leg. 3, $\mathrm{n}^{\circ} 14 ;$ MDbI, $\mathrm{n}^{\circ} 374$, p. 395; Miquel, $\mathrm{n}^{\circ}$ 58, p.45. 'Ex speciali dilectionis' $M D h I, \mathrm{n}^{0} 382$, p. 399; Potthast, 3306-7; PL, 215, 1342 . (Without incipit) ACA, Butlles, Innocent III, leg. 3, $\mathrm{n}^{\circ} 16 ; M D b I, \mathrm{n}^{\circ} 383$, pp. 400; Miquel, $\mathrm{n}^{\circ}$ 61, p. 46. 'Ut Carissimus', $M D b I, \mathrm{n}^{\circ} 389$, pp. 403-404; Potthast, 3485; PL, 215, 1449.

29 'Cum ad optinendam' (16/6/1205), $M D b I, \mathrm{n}^{\circ} 318$, p. 349; Potthast, 2544; PL, 215, 666.

30 'Quanta bona' (16/6/1205), ACA, Butiles, Innocent III, leg. 3, $\mathrm{n}^{\circ} 11 ; M D h I, \mathbf{n}^{\circ} 316$, p. 347; Miquel, $\mathbf{n}^{\circ} 55$, p. 44

31 'Proposuic coram' (16/6/1205), MDbI, $\mathrm{n}^{0}$ 321, p. 351; Potthast, 2558; PL, 215, 666.

32 ACA, Butlles, Innocent III, leg. 3, $\mathrm{n}^{\circ} 7 ; M D b I, \mathrm{n}^{\circ} 306$, p. 339; Miquel, $\mathrm{n}^{0}$ 51, p. 42; FrIED, Päpstliche Schutz, p. 329. The Annali Genovesi di Caffaro e de'suoi sontinuatori, ed. L. BELGRANO and Cesare IMPERIALE Di SANT'ANGelo, 5 vols, I890-1929, 2, p. 94, brevemente recogida la visita de Pedro: 'In isto vero anno intravit Ianuam rex Aragonensis'.

33 Liber Maiolichinus de gestis Pisanorum Illustribus, ed. C. CALISSE, Rome 1904; Codice diplomatico della Repubblica di Genova, 3 vols, ed. C. Imperiale di Sant'Angelo, Rome 1942, 3, nos. 52-53, pp. 137-140; MIreT, uItinerariom, 3, PP. 156-157; ACA, perg. Pere I, 188; Colectión de 
Pero financiera y militarmente el proyecto iba más allá de las intenciones de Pedro y nunca se llevó a cabo ${ }^{34}$. Inocencio, aunque ofreció su ayuda, ya le había avisado, en febrero de 1204, de que dada la fuerza de los almohades y las divisiones de los cristianos, un ataque victorioso sería improbable ${ }^{35}$. Sin embargo, fue pedida una importante ayuda internacional a los genoveses, difícilmente influenciados por una coronación.

2.1.3. Para incrementar su reputación en casa. Mientras hay evidencia poco concreta de que Pedro estuviera intentando vencer a otros gobernantes ibéricos ${ }^{36}$, pudo haber sido influenciado por problemas internos. Debido a un periodo de gran expansión en la primera mitad del siglo XII, seguido por un periodo difícil después de la aparición de los almohades, la Corona luchó contra una vieja nobleza desposeída de poder por el cambio de la frontera, y contra otra nueva nobleza en el sur, carente de una válvula de escape belicosa ${ }^{37}$. Estos sectores de la nobleza alcanzaron el culmen de su descontento en los años de su coronación. Ante la ausencia de un éxito militar, la Corona pudo haber deseado refor-

documentos inéditos del Archito de la Corona de Aragón fCDIACA), 8, pp. 95-96; DOXEY, Gary, «Diplomacy, trade and war: Muslim Majorca in international politics, $1159-1181$ " Joumal of Medietal History 20 (1994) 39-61; ABULAFlA, A Mediterranean Emporium: The Catalan Kingdom of Majora, Cambridge 1994, pp. 3-7; CABestany, Joan, "Alfons el Cast": en Els Primers Comtes-Reis: Ramon Berenguer IV, Alfons el Cast, Pere El Catolic, por P. SCHRAMM, J. CABESTANY, E. BaGUÉ, Barceiona 1963, p. 79; PALACIOS MARTí, La Caronación, pp. 39-41; ViCENS VIVES, Jaime, Manual de Historia Económica de España, Barcelona 1959, p. 151. Para la invasión musulmana de 1203, ver el excelente estudio de HUICI MIRANDA, Ambrosio, Historia politica del imperio Almobade, 2 vols, Teruán 19561957, 2, pp. 397-399.

34 Bisson, Conservation of Coinage: Monetary Exploitation and its Restraint in France, Catalonia, and Aragon (c. A.D. 1000- c.1225), Oxford 1979, p. 97, describe el proyecto de la conquista Balear como la única de las propuestas de Pedro, que era más que un «quiero y no puedo».

${ }^{35}$ Register 6., $\mathrm{n}^{\circ} 234$ (235), pp. 395-396; MDhl, $\mathrm{n}^{\circ}$ 295, pp. 329-30; Potthast, 2127; PL, 215 , 265.

36 LINEHAN, (History and the Historians, p. 570) ha sugerido que el propósito de la coronación puede haber sido «sorprender a los reyes de Castilla y León». Mientras hay mucha probabilidad de que Pedro hubiera deseado acrecentar su reputación en la Península, hay pocos datos que indiquen las circunstancias precisas en que esto pudiera haber liegado a ser deseable. El último gran movimiento ceremonial habia tenido lugar cuando Alfonso VIII armó caballero a Alfonso IX en 1188 (GonZÁLEz, Julio, El reino de Castilla en la época de Alfonso VIII, 3 vols. Madrid 1960, 1, pp. 705-706; GonZÁlez, Julio Alfonso IX, 2 vols., Madrid 1944, p. 55; JIMÉNEZ DE RADA, Rodrigo, Historia de Rebes Hispanie, VII, c. 24, p. 247). Los reyes de Castilla, León y Portugal, no habian sido coronados, y en Navarra ellos continuaban con la práctica de elevar a su rey sobre un escudo (SCHRAMM, «Der Köning von Navarra (1035-1512)*: Zeitschrift der Sabigny-Stiftung für Rechtsgeschicbte 81 (1951) 150.

37 SALRACH, José, Història de Catalunya, II: El Procés de Feudalització, Barcelona 1987, pp. 361398; FReEdman, Paul, The Origins of peasant servitude in medieval Catalonia, Cambridge 1991, pp. 110-116; FreEDMan, "The Catalan Ius Maletractandi»: en Recueil de Mémoires et Travaux publié par la Société d Histoire du Droit et des institutions des Anciens Pays de Droit Écrit, 13 (1985) 40; BISSON, The Medieval Crown of Aragon, Oxford 1986, pp. 38-39; BIsson, «Some characteristics of Mediterranean tercicorial powers: Proceedings of the American Philosopbical Society 123 (1975) 149; Busson, "The «Feudal Revolutionm": Past and Present 142 (1994) 33-34. 
zar su sagrada legitimidad. El compromiso de «Cum quanta gloria» (16/6/1205) en el cual Inocencio ordenaba que, después de pedir la corona de Roma, futuras coronaciones tuvieran lugar en Zaragoza — pero llevadas a cabo por el Arzobispo de Tarragona-, puede indicar que fuera imposible que la coronación se efectuara «en casa» $\sin$ inquietar a los catalanes o a los aragoneses o bien a ambos ${ }^{38}$.

2.1.4. La herejía albigense: por la protección papal contra la expansión capeta; para Pedro proponerse a sí mismo como campeón de la ortodoxia; para exaltar su posición ante los señores del sur de Francia. Tres cartas papales de junio de 1205, dos concediendo a Pedro posesión de tietras capturadas a los herejes ${ }^{39}$, y la tercera urgiendo a los eclesiásticos a ayudar a Pedro a expulsar$\operatorname{los}^{40}$, indican que el problema fue tratado en Roma. Los lazos geográficos, económicos, demográficos, religiosos y culturales de Aragón con el Mediodía eran estrechos ${ }^{41}$. Políticamente Aragón se había constituido en el primer poder de la región. Puede objetarse que en 1204 no había cruzada ni expansión capeta. Pero ambos, Inocencio y Pedro, tenían sus piezas en posición para el juego mucho antes de 1209 . Inocencio había enviado legados ${ }^{42}$, había pedido la colaboración de Felipe Augusto para una Cruzada ${ }^{43}$, y había despojado de sus oficios a Arzobispos incompetentes, principalmente al tío de Pedro, el Arzobispo Berenguer de Narbona ${ }^{44}$. En 1198 y otra vez en febrero de 1204 Pedro publicó severos edictos contra la herejía ${ }^{45}$. El viaje de Pedro a Roma fue financiado en gran parte por su cuñado Raimundo VI de Tolosa ${ }^{46}$.

${ }_{38}$ MDbl, n ${ }^{\circ} 314$, pp. 346 347; Potthast, 2543; PL, 215, 665

39 'Cum ad expellendam' $M D h I, \mathrm{n}^{\circ} 319$, pp. 349-350; Pottbast, 2545; PL, 215, 666. 'Discretioni vestre' MDhI, $\mathrm{n}^{\circ} 322$, pp. 351-352; PL, 215, 667; Potthast, 2540.

40 'Cum carissimus' $M D h I, \mathrm{n}^{\circ} 320$, p. 350; Potthast, 2546; PL, 215, 666.

4. La literatura sobre este tema es amplia. Ver VICENS VIVES, Manual de Historia, pp. 141-142; Ventura, Alfons 'El Cast', Barcelona 1961, p. 6; ABADAL I DE VINYALS, "À propos de la "domination" de la maison comtale de Barcelone sur le Midi français»: Annales du Midi 76 (1964), 315-345; BONNASSIE, «L 'Occitanie, Un État manqué?: L'Hissoire 14 (1979) 31-40; DefourNEAUX, M., Les Frangais en Espagne aux XIe et XIle siècles, Paris 1949; HiGOUNET, Charles, «Un mapa de las relaciones monásticas transpirenaicas en la Edad Median: Pirineos 7 (1951) 543-544; HIGOUNET, «Un grand chapitre de l'histoire du XIIe siècle: la rivalité des maisons de Toulouse et de Barcelone pour la prépondêrance méridionale»: Mélanges Louis Halpben, Paris 1951, 315; MuNDó, Anscari, "Moissac, Cluny ex les mouvements monastiques de L'Est des Pyrénées du Xe au XIIe siècle»: Annales $d u$ Midi 75 (1963) 551-573; DAVID, Pierre, Études Historiques sur la Galice et le Portugal du Vie au XIle Siécle, Lisbon-Paris 1947, esp. pp.341-439; Gouron, André, "Aux origines de l'influence des glossareurs en Espagne»: Historia, Instituciones, Documentos 10 (1983) 325-346.

42 Potthast, 764, PL, 214, 675-6; Potthast, 2230, PL, 215, 360.

13 Potthast, 2009, PL, 215, 176-80; Potthast, 2225, PL, 215, 361.

14 Potthast, 169 (PL, 214, 142), 503 (214, 457-458), 1177 (214, 903-906), 1928 (215, 8384), $2103(215,273-274), 2129$ (215, 272), $2224(215,355-357), 2238(215,368)$,

is (1198) MaRCA, Pierre de, Marca bispanica sive limes bispanicus, boc est geographica et bistorica descriptio Catalonit, Ruscinonis et circumdiacentizm populorum, Paris 1688, p. 517; MIRET, “Itinerario", 3, pp. 85, 153; VenTURA, Pere el Catolic, p.50. (1204) MIRET, «Itinerario", 3, pp. 274-283; IBARRA 
2.1.5. Para asegurar el poder de Aragón y la independencia del Imperio en Provenza. La mayoría de los mencionados por el Ordo como acompañantes de Pedro son figuras importantes en la política provenzal ${ }^{47}$. Alfonso II, padre de Pedro, gastó mucha energía vigorizando el control de Aragón en las ciudades de la región, particularmente en Atles, Niza y Marsella ${ }^{48}$. Teóricamente Aragón detentaba el Condado de Provenza en feudo de parte del Emperador ${ }^{49}$, pero Alfonso II había mostrado un espíritu desafiante, rehusando asistir a la coronación Burgundia de Barbarroja en $1178^{50}$, y aconsejando a su hermano el conde Ramón Berenguer de Provenza que evitara rendir homenaje al Emperador $^{51}$. Las relaciones entre Aragón y el Imperio empeoraron hasta el punto de que Enrique VI pidió a los perplejos genoveses invadir la corona de Aragón ${ }^{52}$. Quizá sacando ventaja del conflicto imperial entre Felipe de Suabia y Otto de Brunswick, Pedro fue ganando protección para sus posesiones provenzales y usó de la coronación para reforzat su situación ante Roma y la nobleza de la región.

2.1.6. Esto es definitivamente lo que Sherlock Holmes habría llamado «un problema de tres pipas». Sin embargo, hay que recalcar un rasgo adicional. En

ORoz, Maria Africa, «Nuevas aportaciones para el itinerario de Pedro el Católicon: CHCA 7 (1962), ii. p. 74; Histoire Générale de Languedoc, 6, p. 231.

46 MIRET, «Itinerario", 3, 274; IBARRA OROZ, "Nuevas aportaciones», p. 74; BISsON, Fiscal Accounts of Catalonia 1151-1213, 2 vols., California 1984, 1, p. 130; Histoire Générale de Languedoc, $8,518-522$.

47 Sobre Sancho, ver Ventura, Alfons el Cast, pp. 206-21 I; SANPERE y MIQUeL, Salvador, "Minoría de Jaime I: vindicación del procurador Conde Sancho»: CHCA 1 (1912), II, pp. 580-594. Sobre Arnau de Castellbò, Ventura, Pere el Catòlic, pp. 51-54; Sobre Hugh de Baux, Higounet, «Un grand chapitre», 318-320. Sobre el Vizconde de Marsella; Aurell i CARDONA, Marti, «L 'expansion Catalane en Provence au XIIe sièclen: La fornació i expansió del feudalisme català, ed. Jaume PORTElla I COMas, Girona 1986, p. 188; Foreville, Raymonde, Le Pape Innocent III et La France, Stuttgart 1992, pp, 63-64, 290. «Parecería que el Abate Guillermo de Bornieux estaría en Roma obteniendo privilegios para su abadía benedictinas (Register 7, $\mathrm{n}^{\circ}$ 162, pp. 278-287).

48 Alfonso Il Rey de Aragón, Conde de Barcelona y Marquếs de Provenza. Documentos (1162-1196), ed. Ana Isabel SánCHEz CaSABón, Zaragoza 1995, en especial (Arles) n ${ }^{\circ} 506$, pp. 669-671, n $^{\circ}$ 629, pp. 820-825, $n^{\circ} 634$, pp. 828-829; (Niza), $n^{0}$ 501, pp. 663-664; (Marsella), $\mathrm{n}^{\circ}$ 590, pp. $765-$ 767.

49 Liber Feudorum Maior, ed. F. MiQUEL ROSEL, 2 vols., Barcelona 1947, 2, nos. 901-902, pp. 366-371.

50 Baratier, Édouard, Histoire de la Provence, Toulouse 1969, p. 142; CabestanY, "Alfons el Cast», 71; HIGOUNET, «Un grand chapitre», 319-20; PALACIOS, La Coronación, p. 35.

st Histoire Générale de Languedoc, 6, p. 33; РACAUT, Marcel, Fréderic Barberousse, Paris 1967, p. 274; AURELL, «L'expansion catalane en Provence», 181; PALACIOS, La Coronación, p. 35.

52 Annali Genovesi, 2, pp.58-9: 'ego consimile habeo, et bene novi quid in eo continetur. vultis vos placitari mecum? ego vobis faciam rationem. set illud vere sciatis, quod non dabo vobis in regno Siciliae, nec eritis mel consortes; nec ad hoc studeatis ut mecum habeatis terram communem; set, si vulcis exercitum facere, ego conferam vobis ad capiendum aliud regnum, quod totum sit vestrum. Si vultis exercirum facere super regem Aragonae, ego ero vobiscum. quid plura?'; Cf. ABULAFIA, The Two Italies, Cambridge 1977, pp. 211-212. 
los primeros años de su reinado, Pedro vio a políticos más viejos e inteligentes usar efectivamente el poder papal, a veces en detrimento suyo. Cuando Pedro trató de arrebatar a su madre, Sancha, los importantes castillos fronterizos que separaban Aragón de Castilla, que le fueron dados por su marido Alfonso II $^{53}$, Sancha contraatacó ganando dos bulas de Inocencio: la primera protegiéndola junto con las posesiones que le dejó Alfonso ${ }^{54}$, y la segunda convocando al Arzobispo de Tarragona y sus votantes para protegerla en tanto que viudass. Al final de la campaña aragoneso-castellana de 1197 en Navarra, Sancho el Craso de Navarra había sido liberado por Inocencio de un juramento en el que había prometido a su hermana Teresa en matrimonio a Pedro, pues tal juramento había sido hecho bajo coacción ${ }^{56}$. El mismo Pedro, habiendo prometido en su ascenso al trono conservar el sistema monetario devaluado durante los postreros años de su padre, fue forzado por el clamor popular a apelar a Inocencio para que le levantara su juramento 57 . En 1204, Pedro, ya un adulto de 27 años, pudo haber sido consciente de obtener algunos beneficios merced al Papado.

53 Sobre la dote de Sancha, ver CDIACA, 4, p. 409; ACA, perg. Alfons I, 146. Sobre las propiedades de Sancha y la disputa con Pedro, ver MIRET, «Itinerariom, 3, p. 83; ZURITA, Anales, 2, p. 133; BAGUÉ, «Pere el Catòlic», 104-5; Ventura, Pere el Catolic, Barcelona 1960, p.52. Sobre la intervención castellana en la disputa, GoNZArez, Alfonso VIII, 1, pp. 844, 856; 3, n ${ }^{\circ} 667$, pp. 17982; ACA, perg. Pere I, 98, 121; CIROT, G., "Appendices (Chronique Latine)»: Bulletin Hispanique 20 (1918) 159-166; MIRer, «Itinerario», 3, pp. 245, 248. El obispo Juan de Osma, en la Cbronique Latine, sugería una relacion impropia entre Sancha y Alfonso VIII (CIROT, «Chronique latine inédite des Rois de Castille (1236)m: Bulletin Hispanique 14 (1912) 262; LOMAX, "The authorship of the Chronique Latine des Rois de Castlle»: Bulletin of Hispanic Studier 40 (1963), 205-211).

54 'Cum a nobis petitur' (13/5/1199), Biblioseca de Catalunya, Arxiu, perg. 3541, 4-VI-8; MDbI, $\mathbf{n}^{\circ}$ 228, p. 260; Pottbast, 1035. cf. Papsturkunden in Spanien, 2 vols, ed. P. KeHR, Berlin 1926,1 , nos. 268-269, pp. 578-579.

5s 'Quod viduarum defensio' ( $5 / 5 / 1200$ ), Biblioceca de Catalunya, Arxiu, perg. 3540, 4-VI-8. of. Papstureunden in Spanien, $1, \mathrm{n}^{\circ} 238$, p. 539, 254, p. 560, 268-269, pp. 578-579; Dacumentas de Sigena, ed. A. Ubieto ARTETA, Valencia 1972 , $\mathrm{n}^{\circ} 17$, p. $49, \mathrm{n}^{\circ} 21$, p. $54-55, \mathrm{n}^{\circ} 24$, p. $57, \mathrm{n}^{\circ} 32$, pp. 67-68.

36 Register 1, $\mathrm{n}^{\circ} 553$ (556), pp. 801-802; MDhI, $\mathrm{n}^{\circ}$ 181, pp. 197-8; Potthast, 597; PL 214, 509. Sobre las ambiciones aragoneso-castellanas en Navarra, ver Liber Feudortum Mayor $1, \mathrm{n}^{\circ} 28$, p$37, \mathrm{n}^{\circ} 29$, p. 39, $\mathrm{n}^{\circ} 33$, p. $47, \mathrm{n}^{\circ} 34$, p. 48; Gonzilez, Alfonso Vlil, 2, $\mathrm{n}^{\circ} 320$, pp. 530-532, 3, $\mathrm{n}^{\circ}$ 667, pp. 179-I86; UBIETo ARTETA, A., "Navarra-Aragón y la idea imperial de Alfonso vII de Castillan: EEMCA 6 (1956) 41-82; sobre la campaña de 1197, ver, RoDrigo, Historia de Rebus Hispanie, chs. 30-32, pp. 252-254; CIROT, "Chronique Latine", c. 16, Pp. 265-266; LuCAS de TúY, Crónica de España, ed. J. PUYOL, Madrid 1926, c. 83, p. 408; Los Anales Toledanos I y II, ed. J. P. Martín Cleto, Toledo 1993, p. 165; Zerbi, P., Papato, Impero, e Res'publica Cbristiana dal 1187 al 1198, Milan 1955, pp. 157-160; LACARRA, José, Historia del reino de Navarra en la edad Media, Pamplona 1975, pp. 228-229; GonZález, «Reclamaciones de Alfonso VIII a Sancho el Fuerte y tratado del reparto de Navarra en 1198*: Hispania 3 (1943) 545-568. Sobre el libramiento de juramentos: MANSlLLA, Demetrio, «Inocencio III y los reinos Hispanos»: Anthologica Annwa 2 (1954) 39; cf. BRYSON, W., «Papal releases from toyal oachs»: Joumal of Ectesiastical History 22 (1971) 19-33.

57 Register 2., $\mathrm{n}^{\circ}$ 28, pp. 42-44: $M D b l, \mathrm{n}^{\circ} 183$, p. 199; Potthast, 656; PL, 214, 558. Sobre ésto, Bisson, Conservation of Coinage, pp. 85-87, pp. 166-77; Bisson, "Quanto personam tuam (X 2. 24. 18): its original significance» en Proceedings of the Fourth International Congress of Medieval Canon 
2.2.1. Cualesquiera que fuesen las intenciones de Pedro, la coronación dependía de las ventajas que tuviese para Inocencio III. La reafirmación de la alianza cerrada con Aragón ${ }^{58}$, la renovación del pago del censo ${ }^{59}$, y la exaltación y protección de un gobernante que expresaba su deseo de luchar contra la herejía y los musulmanes, también beneficiaba al Papado ${ }^{60}$. Pero Inocencio no estaba pensando precisamente en los problemas aragoneses.

2.2.2. Está claro, por el peso de la correspondencia respecto al problema, que Inocencio consideraba de primera importancia el matrimonio de Federico de Sicilia con una novia aragonesa ${ }^{61}$. En agosto de 1204 , Inocencio mandó a Pedro ir directamente a Roma, antes que ir a Génova y Pisa, de manera que

Law, ed. Stephan KUTTNER, Vaticano 1976, pp. 229-49; cf. Papsturkunden in Spanien, 1, $\mathrm{n}^{\circ} 238$, p. 539;JACA: Documentos Municipales $971-1269$, ed. A. UBIETO ARTETA, Valencia 1975, ${ }^{\circ} 25$, p. 77 ; El Libro de la Cadena del Concejo de JACA, ed. D. SANGorRín, Zaragoza 1979, n² 24, pp. 211-214; MireT, «Itinerario», 3, pp. 86-87.

58 See FuEd, Päpstliche Schutz, pp. 53-103, 184-205; KEHR, Das Papsttum und der katalanische Prinzipat bis zur Vereinigung mit Aragon, Berlin 1926; KeHR, «Cómo y cuándo", pp. 285-326; KEHR, «El Papado y los reinos de Navarra y Aragón hasta mediados del siglo XII", pp. 74-186; ABADAL, "L'esprit de Cluny», pp. 3-41; BONNASSIE, La Catalogne, 1, pp. 332-7; 2, pp. 939-42.

59 Sobre el pago original del censo por Sáncho Ramírez, SOMERVILle, Robere, Pope Urban $I$, The Collectio Britannica, and the Council of Melfi (1089), Oxford 1996, pp. 97-8, 157-8; Regesta pontificum romanorum, ed. P. JAFFÉ, rev. S. LOEWENFELD, Leipzig $1885 U L \mathrm{~J}$, 5398-9, Sobre la renovación del censo por Pedro I, $M D b 1, \mathrm{n}^{\circ} 34$, p. 53; $J L$, 5552; Liber Feudorum Maior, $1, \mathrm{n}^{\circ} 4$, pp. 6-8; Colección diplomática de Pedro I de Aragón y Navarra, ed. A. UBIETO ARTETA, Zaragoza 1951, $\mathrm{n}^{\circ} 21$, p.235. Para el pago del censo en 1098, MDhI, $\mathrm{n}^{\circ} 39$, pp. 58-9; VILlanueva, Joaquín, Viage literario a las iglesias de España, 22 vols., Madrid 1803-1852 [VL], 15, p. 361; Colectión diplomática de Pedro $I, \mathrm{n}^{\circ} 58$, p. 292. Es digno de atención que en los últimos años del siglo XII, mientras el cardenal Albino anotaba en la lista de su censo a regnum Aragone iuris beati Petri est, D auri mancusios ad cuneum Jacce singulis annis", CenNIO (el posterior Honorio III) no menciona el censo aragonés en sus cuentas en absoluto (Le Liber Censuum de L'Église Romaine, 3 vols, ed. P. Fabre, L. Duchesne, G. Mollat, Paris 1889-1952, 2, p. 107; Palacios, La Coronación, p. 50).

60 Sobre Pedro II e Inocencio III en la cruzada Albiguense, SMiTH, Damian, Innocent III and Aragon-Catalonia: Studies in Papal power, PhD thesis, Department of Medieval History, University of Birmingham 1997, pp. 94-168. Sobre el papado y la cruzada balear, GoŃI GAZTAMBIDE, José, Historia de la Bula de la Cruzada en España, Vitoria 1958, pp. 68-70; FERrER, Miguel, «Mallorca y la teocracia pontifician: Analecta Sacra Tarraconensia 23 (1950) 16.

${ }_{61}$ 'Ad tuam volumus' (5/6/1202) MDbl, n² 261, pp. 282-283, Potthast, 1698, PL, 214, 1018. 'Gaudemus in Domino' (8/8/1204) ACA, Butlles Innocent III, leg. 3, no 7, MDbl, n ${ }^{\circ} 306$, p. 339 , Miquel, $\mathbf{n}^{\circ}$ 51, p. 42, Fried , Päpstliche Scbutz, p. 329. 'Dilectum Filium' (24/11/1204) ACA, Butlles, Innocent III, leg. $3, \mathrm{n}^{\circ} 8, M D h I, \mathrm{n}^{\circ} 308$, pp. 341-342, Miquel, $\mathrm{n}^{\circ} 52$, p. 43 . 'Paulo antequam' (27/1/1207) MDbI, no 359, p. 382, Potthast, n' 2992, PL, 215, 1082; 'Super negotio' (2/11/1207) ACA, Butlles, Innocent III, leg. 3, $n^{\circ} 14, M D b I, n^{\circ} 374$, p. 395, Miquel, $n^{\circ} 58$, p.45. 'Ex speciali dilectionis' (25-27/2/1208) MDbl, $\mathrm{n}^{\circ}$ 382, p. 398, Potthast, 3306-7, Miquel, $\mathrm{n}^{\circ}$ 59, p. 45; 'Damaged bull without incipit' (19/4/1208) ACA, Butlles, leg. 3, $\mathrm{n}^{\circ} 16, M D b I, \mathrm{n}^{\circ} 383$, p. 400, Miquel, $\mathrm{n}^{\circ} 61$, p. 46 . 'Ut Carissimus' (8/8/1208) MDhI, $\mathrm{n}^{\circ} 389$, pp.403-404, Potthast, 3485, PL, 215, 1449. 'Gaudemus in Domino' (28/3/1209) ACA, Butlles, Innocent III, leg. 3, no 17, MDbI, no 399, pp. 416-7, Miquel, $\mathrm{n}^{\circ} 61$, p. 46.

Hispania, $\mathrm{L} X / 1$, núm. 204 (2000) 163-179 
pudiera negociar directamente con los enviados de Federico ${ }^{62}$. El aumento de la influencia papal en el reino de Sicilia, la protección de su pupilo y la destrucción de las ambiciones observadas en Felipe de Suabia eran problemas de gran importancia para Inocencio. Una novia para Federico de una dinastía leal a la Santa Sede, la oferta de ayuda militar y financiera, eran cosas muy apreciadas por Inocencio $\mathrm{III}^{63}$. La coronación podía impresionar a los mensajeros de Federico con el estatus de Pedro.

2.2.3. Libertas ecclesiae. Aunque el estatuto de Pedro concediendo la libertad de elecciones eclesiásticas está fechado por la chancillería el 30 de octubre de $1207^{64}$, es probable que esto fuera acordado durante la coronación ${ }^{65}$. La Gesta Comitum Barcinonensium estaba segura de ello ${ }^{66}$. Quizá el acuerdo se mantuvo supuestamente en secreto hasta que Pedro pudiera anunciarlo en un momento políticamente conveniente. Se supone que para Pedro la libertad de la Iglesia suponía poco y no alteraba mucho sus relaciones con la Iglesia. Para Inocencio III, que de joven había ido a Inglaterra a visitar la tumba de Becket en Canterbury, éste fue un objetivo apreciado ${ }^{67}$.

2.2.4. Roma. No deberíamos dejar de considerar la posición de Inocencio en Roma en 1203-4. Las pretensiones papales ante el mundo estaban frecuentemente emparejadas con crisis domésticas. La Curia percibía que los feudos de los nobles romanos estaban llevando a Roma hacia una guerra civil68. En mayo de 1203 Inocencio había abandonado Roma, volviendo nueve meses después ${ }^{69}$.

62 'Gaudemus in Domino' (8/8/1204) ACA, Butles, Innocent IIl, leg. 3, n 7, MDbI, n' 306, p. 339, Miquel, no. 51, p. 42, FRed, Päpstliche Schutz, p. 329.

${ }^{6}$ Gesta Innocentii, passim; ROBINSON, Ian, The Papacy 1073-1198: Continuity and Innovation, Cambridge 1990, pp. 367-524; WALEY, Daniel, The Papal State in the Thirteenth Century, London 1961, p. 26; KENNAN, Elizabeth, «Innocent III and the First Political Crusade»: Traditio 27 (1971) 232-233; Abulafia, Frederick II, p. 85; TillmanN, Helena, Pope Innocent III, North-Holland 1980, pp. 103-187; ParTner, Peter, The Lands of St Peter: The Papal State in the Middle Ages and the Early Renaissance, London 1972, p. 231.

64 MDbl, $\mathrm{n}^{\circ} 373$, pp. 394 395, Potchast, 3207, PL, $215,1243$.

65 ENGels, Odilo, "Privilegios de Pedro el Católico en favor de obispos Catalanes": CHCA 7 (1962), III, pp. 37.39.

66 Gesza Comizum Barcinonensitum, c. 25, p. 51 : 'Quo facto, dominus rex Petrus sua mera liberalitate et voluntate ob honorem Dei et Romanae ecclesiae ac dornini papae Innocentii, dedit et fecit libertatem omnibus ecclesiis et monasteriis regni sui quod sine sui consilio et suorum possent libere eligere in suis ecclesiis, cum vacarent; nam antea sine consensu regis in cathedralibus ecclesiis et monasteriis eligere non audebant'.

67 Sobre Inocencio III, Pedro II y Libertas Esclesiate ver SMITH, Innosent III and Aragon-Catalonia, pp. 234-272. Sobre Inocencio en Canterbury, Maccarrone, Michele, "Innocenzo lil Prima del Pontificaton: Arcbivio della Societa Romana di Storia Patria 66 (1943), 78; MOORE, John, «Lotario dei Conti di Segni (Pope Innocent III) in the 1180s»: Ambrizum Historiae Pontificiae 29 (1991) 255-258.

68 WALEY, The Papal State, Pp.45-6; TILLMANN, Innocent II, p.128; PARTNER, The Lands of $S t$ Peter, p. 239; RoBINSON, The Papacy, pp. 1-32.

69 Gesta Innocentii, chs. 135-7 in PL, 214, 182-7; Waley, The Papal State, p. 45; TillmaNn, Innocent IIl, pp. 128-129. 
De acuerdo con la Gesta Innocentii, la violencia incontrolada y la pérdida de litigantes para la corte papal dejó su marca sobre el pueblo romano ${ }^{70}$. Inocencio emprendió la restauración del propio prestigio papal en Roma. Esto fue hecho parcialmente a través de una serie de perspicaces nombramientos administrativos ${ }^{71}$, pero Inocencio era consciente de los beneficios de un gran espectáculo público, otro "espectáculo con un significado", un mensaje visible de cómo el Papado acrecentaba la dignidad de Roma ${ }^{72}$.

2.2.5. El Imperio. Hacia 1204, Felipe de Suabia había ganado ascendiente en el conflicto imperial ${ }^{73}$. Pero las preferencias de Inocencio para candidato imperial dependían parcialmente de cual candidato había sido coronado propiamente como Rey ${ }^{74}$. Felipe había sido coronado en un momento incorrecto, en el lugar incorrecto y también por el Arzobispo incorrecto ${ }^{75}$. La coronación de Pedro II, como la de Johanittsa de Bulgaria por el Cardenal Leo Brancaleone hacia el mismo tiempo ${ }^{76}$, puede ser entendida como parte de una guerra de propaganda, una demostración del cómo, dónde y por quién de las coronaciones. Sin embargo la coronación de un Rey en Roma, en forma similar a la de la coronación Imperial ${ }^{77}$, podía sugerir que había otros que podían disputar la elevada posición de Emperador.

70 Gesta Innocentii, PL, 214, 189; TILlMANN, Innocent III, p.129; WALEY, The Papal Staze, p. 46.

1 Gesta Innocentii, PL, 214, 189; TIllmann, Innocent III, p.129; PARTNER, The Lands of St Peter, p. 239; WALEY, The Papal State, pp.46-7. Sobre la importancia de Roma para Inocencio III ver BOLTON, Brenda, «Rome as a setting for God's grace»: Innocent III: Studies on papal authority and pastoral care, Variorum 1995, pp.1-17; MOORE, J., "The Sermons of Pope Innocent IIl": Römische Historische Mitteilungen 36 (1994) 108.

72 Cf. BOLTON, "A show with a meaning: Innocent's approach to the Fourth Lateran Council,1215": Medieval History 1 (1991) 53-67.

${ }_{73}$ KEMPF, Friedrich, Papsttum und Kaisertum bei Innocenz III, Rome 1954, esp. pp. 1-64; TILIMANN, Innocent III, p. 124; WALEY, The Papal State, pp. 48-49.

${ }^{74}$ Regestum Innocentii III papae super negotio romani imperii, ed. F. KEMPF, Roma 1947, ${ }^{\circ} 62$, p. 168; Pottbast, 1653; KEMPF, Papsttum und Kaisertum, esp. pp. 48-55; KEMPF, «Innocent III und der deutsche Thronstreit»: Arcbivum Historiae Pontificiae 23 (1985) 78; MACCARRONE, Chiesa e Stato nella dottrina di Papa Innocenzo III, Roma 1940, pp. 133-42; WATT, Jack, «The Theory of Papal Monarchy": Traditio 20 (1964) 212-213; FuHRMANN, H.,. «Zur bulle Venerabilenn: Historiscbe Forscbungen fit W. Scblesinger, 2, Cologne 1974, 514-517; TIERNEY, Brian, The Crisis of Church and State 1050-1300, New Jersey 1964, p.129.

7s Al menos así es como lo vio Adolfo el arzobispo de Colonia. Felipe fue coronado en Maguncia (Otto de Brunswick fue coronado correctarnente en Aquisgrán), el 8 de septiembre de 1198 (Otto fue coronado el 12 de julio de 1198), y por el arzobispo Aimo de Tarentaise (Otto fue coronado por el arzobispo Adolfo). Ver KEMPF, «Innocent III und der deutsche Thronstreit», p. 65.

76 Register 7, nos. 230-1, pp. 409-412; SWEENEY, James, «Innocent III, Hungary and the Bulgarian Coronations: Church Histary 42 (1973) 320-334.

77 La concesión del orbe a Pedro no pudo sino evocar recuerdos de la concesión del mismo sím. bolo por Celestino III a Enrique VI el 15 de abril de 1191 y las complicaciones que a ello siguieron. Ver Perrun, C.-E., «Les Négociations de 1196 entre L 'Empereur Henri VI et le Pape Célestin III": 
3.1. Las consecuencias políticas de los acontecimientos de 1204 fueron enormes para Aragón. Es muy probable que las extensas revueltas de 1205 en Aragón y Cataluña, mientras que eran relacionadas con lo que se veía como abusos reales en los impuestos, la administración y la justicia, fueran en parte las chispas lanzadas por la coronación y la concesión del censo, y posiblemente por tumores sobre la libertad de la Iglesia, respecto a todo lo cual, casi con certeza los nobles aragoneses nunca habían sido consultados ${ }^{78}$. En la cruzada albiguense, el Papa y los legados papales en el Languedoc consideraron que a causa de los favores otorgados a Pedro a través de la coronación y del matrimonio siciliano, Pedro no debería ni podía intervenir en defensa de los señores franceses del sur de Francia ${ }^{79}$. En la parte aragonesa, según avanzaba Monfort, los beneficios de la protección papal, parecían quedar en cierto modo

Mélanges Louis Halphen, Paris 1951, 566; Jordan, E., «Henri VI a-t-il offert à Celestin III de lui faite hommage pour l'Empire?": Mélanger Ferdinand Lat, Paris 1925, 285-306; ZERBI, P., "Un momento oscuro nella incoronazione romano di Enrico VI (a. 1191)m: Miscellanea Giulio Belvederi, Roma 1954, 517-528. Precisamente en esta época, canonistas ingleses y españoles en especial estaban proponiendo argumentos que reflejaran las nuevas realidades políticas del siglo XIII. Ver POST, Gaines, ««Blessed Lady Spain» - Vincentius Hispanus and Spanish National Imperialism in the Thirteenth Century»: Speculum 29 (1954) 198-209; TIERNEY, Religion, law, and the growth of constitutional thought, Cambridge 1982, p. 22; TIERNEY, «Some recent works on the political theories of the medieval canonists": Traditio 10 (1954) 619; cf. STICKLER, A., "Concerning the political theories of the medieval canonists»: Traditio 7 (1949-1951) 455-457. No todos los reyes se sometian al Emperador y en "Per Venerabilem» Inocencio sugirió que el rey de Francia no conocía autoridad temporal más alta. Ver KeMPF, Papsttum und Kaisertum, pp. 256-262; TIERNEY, "Tria quippe distinguit iudicia...: Una noca sobre el decreto "Per Venerabilem» de Inocencio III: Speculum 37 (1962) 48-59; PENNINGTON, Kenneth, «Pope Innocent III's views on Church and State: a gloss to Per Venerabilem»: en Law, Church and Society: Essays in Honor of Stephan Kuttner, ed. K. PENNINGTON and R. Somerville, University of Pennsylvania Press 1977, pp. 49-67; WATt, "Theory of Papal Monarchy", pp.214-216; MOCHI ONORY, S., Fonti canonistiche dellidea moderna dello stato, Milan 1951, p. 271 ; CHENEY, Christopher., Innocent III and England, Stuttgart 1976, pp. 332-337.

${ }_{78}$ Sobre las revueltas en Cataluña que culminaron en la "carta Magna" de marzo de 1205 , Cartoral dit de Carlemany del bisbe de Girona (s. IX-XIV, ed. J. MARQUÈs, Barcelona I993, 2, $\mathrm{n}^{\circ} 383$, pp. 571-572; BoteT Y SIsó, J., «Cartoral de Carles Many»: BRABLB 4 (1908) n 346, p.322; VILLANUEVA, Viage Literario, I3, p. 158; BIsson, "An 'Unkown Charter' for Catalonia (A.D.1205)»: Album Elemér Mályusz Székesfébêrvar -Budapest 1972 Brussels: Études présentées à la commission Internationale pour ItHistoire des Assemblees des Etats 16 (1976) 61-76; Bisson, Conservation of Coinage, p. 88; Bisson, «L'Essor de la Catalogne: Identité, pouvoir et idéologie dans une société du XIIe siècle»: Annales 39 (1984) 471; BISSON, «Sur les origines du monedatge: Quelques textes inédits»: Annales du Midi 85 (1973) 94-95, 100-101; BIssoN, wPrelude to Power: Kingship and Constitution in the Realms of Aragon, 1175-1250m: The Worlds of Alfonso the Learned and James the Conqueror, ed. Robert BuRNs, Princeton University 1985 , p. 30. Sobre la revuelta aragonesa después de la imposición del "monedaje" en Huesca en noviembre de 1205, ver ZURUTA, Anales, 2, p. 144; Mirer, aItinerario", 3, p. 373; BISSON, «Sur les origines du monedatgen, 91-104; Bisson, Conservation of Coinage, p. 84; BISSON, "Quanto personam tuam», 235; BISSON, «Prelude to Power», 31-32.

79 Para los prelados, MDbI, $\mathrm{n}^{\circ}$ 495, pp. 528-531; PL, 216, 840; Mansi, 22, 886; PIERRE VauX-De-Cennay, Histoire Albigeoise, trad. Pascal Guébin and Henri MaIsonneuve, Paris 1951, chs 377-384, pp. 147-150; SMTH, Innocent III and Aragon-Catalonia, p.142. Para el Papa: MDbI, ${ }^{\circ}$ 
beneficios de la protección papal, parecían quedar en cierto modo lejos de las expectativas $^{80}$. Tras la muerte de Pedro en Muret en 1213, durante la minoría de Jaime I, la protección de 1204, junto con la obligación de ayudar a miserabiles personae, el último testamento de la mujer de Pedro, María de Montpellier, y la petición de los aragoneses solicitando la intervención de Inocencio, formaron la amplia base mediante la cual Inocencio, y su legado, el Cardenal Pedro de Benevento obtuvieron un dominio sin precedentes en Aragón, ampliando la influencia papal en el dominio tempotal hasta el punto de subir los impuestos y redactar leyes ${ }^{81}$.

3.2. Sin embargo, la coronación y el «Cum quanta gloria» habían de tener significado para el resto de los Condes-Reyes: conscientemente todo gobernante aragonés se aseguraba de que si la coronación hubiera de llevarse a cabo, debía hacerse de una manera diferente de la estipulada por Inocencio III -era una cuestión de soberanía. Jaime I (1213-1276) se negó a ser coronado según las condiciones de Gregorio $X^{82}$, pero Pedro III (1276-1285) fue, como todos sus sucesores, coronado sin autorización papal. Es más: Pedro III fue coronado en Zaragoza, y especificó que la coronación fuera en Zaragoza sólo porque era la capital de su reino ${ }^{83}$. Alfonso III (1285-1291) fue coronado por el obispo de Huesca, insistiendo en que su poder no procedía de la Iglesia Romana ni de la Iglesia en general, ni tampoco estaba en contra de la Iglesia ${ }^{84}$. Jaime II (1291-

505, pp. 546-550; Potthast, 4741; PL, 216, 849; Mansi, 22, 878; VAUX-DE-CERNAY, Histoire Albigeoise, chs. 401-41 1, pp. 156-160; SMITH, Innocent III and Aragon-Catalonia, p. 150.

so Ver Ventura, Pere El Catòlic, pp. 193-207; SMITH, Innocent III and Aragon-Catalonia, pp. 137-151.

81 SMITH, D., «Innocent III and the Minority of James I of Aragon»: Anuario de Estudios Medievales (2000), en preparación; VentuRA, Pere El Catolitic, pp. 233-260; SANPERE Y MiQuEL, «Minoria de Jaime I", pp. 580-694; Soldevn A, Ferran, Els Primers Temps de Jaume I, Barcelona 1962.

${ }_{82}$ JAUMe I, Crìnica o Libre dels Feits, ed. F. Soldevila, Barcelona 1982, chs. 536-538, pp. 406-407; BuRNs, Robert, «The spiritual life of James the Conqueror": The Catbolic Historical Review 62 (1976) I5; PALACIOS, La Coronación, Pp. 79-81; PaLACIOS, aLa bula de Inocencio HI y la coronación de los reyes de Aragón»; Hispania 29 (1969) 487; FrIED, Päpstliche Schutz, pp. 250-25 l; PALA. CIOS, «Los actos de coronación y el proceso de usecularización» de la monarquia catalanoaragonesan; État et Église dans la genèse de L'État moderne, Madrid 1986, p. 120; PALACIOS. "Los Símbolos de la Soberanía en la Edad Media española: El simbolismo de la espadan: VII Centenario del Infante D. Fernando de la Cerda: Jornadas de estudio, Ciudad Real, abril 1975; Ponencias y comunicaciones, Madrid 1976, pp. 274-275. En 1229 Gregorio IX había recibido una embajada aragonesa que expresaba el deseo de Jaime de ser coronado por el papa (aunque sin mención de la renovación del censo o del enfeudamiento. Ver Miquel, $\mathrm{n}^{\circ}$ 89, p. 60; FrIED, Päpstliche Schutz, p. 242.

${ }_{33}$ ACA, perg. Pere II, $n^{\circ} 15$; Bernat Desclot, Cromica, ed. M. COLl I Allentorn, Barcelona 1982, c. 73, p. 145; BLANCAS, Coronaciones, pp. 17-18; PALAC1OS, La Coronación, pp. 94-105, 3034; PALACiOS, "La bula de Inocencio III», pp. 490-1; FRIED, Päpstliche Scbutz, p. 251.

si ACA, perg. Alfons II, $n^{\circ}$ 64; ACA. Canc. Reg. 66, fol. 16v; PalACiOS, La Comnación, pp. 114-30, 307-8; PALACIOS, «La bula de Inocencio III", p. 494; BLANCAS, Coronaciones, pp. 22-23; FrIED, Päpstliche Schutz, p. 2S2; PAlacios, "Los actos de coronación», p. 121. 
1327) fue sólo ungido y coronado como Rey de Sicilia ${ }^{85}$, habiendo admitido los catalanes para entonces la oferta de Inocencio III de intervenir en el reino. Alfonso IV (1327-1336) y Pedro IV (1336-1387) colocaron la corona sobre sus propias cabezas ${ }^{86}$. Aunque Pedro IV aceptaba que el metropolitano debía ungir al Rey, destacó que la coronación temporal era el acto constitutivo que mostraba el dominio del príncipe sobre su pueblo ${ }^{87}$.

3.3. Para el Papado, la coronación de Pedro II aparece no sólo como la culminación del proceso por el cual fue ganada la ascendencia sobre AragónCataluña, sino también como el momento en el cual el Papado alcanza la cumbre de su habilidad para sujetar a un gobernante secular a su concepto de sus funciones. Pero justo cuando la gloria de Inocencio III parecía tan particularmente manifiesta, la coronación de Pedro actúa como un recordatorio de la fragilidad del poder político de Inocencio. Pues fue el mismo Rey que fue ungido en Roma y coronado por el Papa, el que concedió la Libertas ecclesiae, ayudó al Papa en Sicilia, legisló contra los herejes con mayor severidad que ningún otro gobernante de su tiempo, y en julio de 1212 ayudó a la derrota de los musulmanes en la decisiva batalla de las Navas de Tolosa ${ }^{88}$; el que murió en Muret por la protección de su tierra y vasallos, luchando con el ejército de una cruzada acometida por el Papa contra la herejía, dirigiendo un ejército en el que todos los miembros excepto el Rey en persona habían sido excomulgados ${ }^{89}$.

\section{Traducción de Leonor Zozaya Montes} y María Zozaya Montes.

\footnotetext{
85 Palacios, La Coronación, Pp. 187-200; Palacios, «La bula de Inocencio III», p. 495.

a6 PaLAClos, La Coronación, pp. 203-269; PaLAClos, «La bula de Inocencio III", pp.497-498; PALACIOS, aLos actos de coronación", p. 121; LINEHAN, History and the Historians, pp. 568-570.

87 PaLACIOS, La Coronación, pp. 229-269; PALACIOS, «La bula de Inocencio III", pp. 499-502.

88 Ventura, Pere El Catolic, pp. 172-184; Huici MiRanda, Las Grandes Batallas, pp. 219327. Sobre el papel de Inocencio III en la campaña, ver SMITH, D., «'Soli Hispani'? Innocent III and Las Navas de Tolosas: Archivum Historiae Pontificiae, 25 pp, en preparación.

89 Por « In privilegio felicis» del 4 de julio de 1213, Inocencio había confirmado la bula de Urbano II del 16 de marzo de 1095 (MDhI, no 34, p. 54; $J L$, 5552; KEHR, Papsturkunden, 2, p. 120; $P L, 216,888$ ), que prohibía a un obispo, arzobispo o legado papal, excomulgar al rey de Aragón o a su mujer sin la orden expresa del Papa.
} 